\title{
Multiple prediction from incomplete data with the focused curvelet transform
}

Felix J. Herrmann*, EOS-UBC, Deli Wang ${ }^{\dagger}$, Jilin University and Gilles Hennenfent ${ }^{*}$, EOS-UBC

\section{SUMMARY}

Incomplete data represents a major challenge for a successful prediction and subsequent removal of multiples. In this paper, a new method will be represented that tackles this challenge in a two-step approach. During the first step, the recenly developed curvelet-based recovery by sparsity-promoting inversion (CRSI) is applied to the data, followed by a prediction of the primaries. During the second high-resolution step, the estimated primaries are used to improve the frequency content of the recovered data by combining the focal transform, defined in terms of the estimated primaries, with the curvelet transform. This focused curvelet transform leads to an improved recovery, which can subsequently be used as input for a second stage of multiple prediction and primary-multiple separation.

\section{INTRODUCTION}

Surface-related multiple prediction and seismic interferometry are examples where weighted multi-dimensional cross-convolutions and cross-correlations of seismic data volumes provide information on Green's functions that describe the Earth response at the surface. For instance, surface-related multiples can approximately be predicted through a weighted multidimensional convolution of the data with itself, while 'daylight imaging' techniques extract the Green's function by cross-correlation of wavefields (see e.g. Wapenaar et al., 2006, which contains a collection of the most recent papers on this topic). Recently, new approaches have been proposed, where the Green's functions are extracted through inversion or deconvolution (See the contributions by Snieder et.al, Schuster et.al. and Berkhout and Verschuur in Wapenaar et al., 2006).

Unfortunately, these multidimensional techniques are sensitive to missing traces (see e.g. Fig. 3(a) where the predicted multiples suffer significantly from the missing data). Many different techniques have been proposed to solve the interpolation problem. The different approaches can roughly be divided into data-dependent approaches, assuming prior (velocity) information on the wave arrivals, and non-parametric approaches that do not make such assumptions. Examples of parametric methods are the so-called data mappings (Bleistein et al., 2001), based on approximate solutions of the wave equation. These methods require information on the seismic velocity. Para- bolic, apex-shifted Radon or migration-like transforms such as DMO/NMO/AMO also fall in this category. Other examples of data-adaptive methods are predictive, dip filtering techniques and plane-wave destructors that require a preprocessing step (see e.g. Spitz, 1999; Fomel and Guitton, 2006). Examples of non-parametric approaches include transform-based sparse inversion methods based on the Fourier or other transforms (Sacchi and Ulrych, 1996; Elad et al., 2005; Zwartjes and Gisolf, 2006; Abma and Kabir, 2006).

In this work, we hold the middle between data-dependent and transform-based methods by combining the dataindependent discrete curvelet transform (FDCT, Candes et al., 2006; Ying et al., 2005; Hennenfent and Herrmann, 2006b) with the recently introduced data-adaptive focal transform (Berkhout and Verschuur, 2006). By virtue of its compression on seismic data and its invariance under wave propagation, the curvelet transform has proven to be an excellent domain for the formulation of seismic processing algorithms ranging from data regularization (Hennenfent and Herrmann, 2006a; Herrmann and Hennenfent, 2007); primary-multiple separation (Herrmann et al., 2007) to migration-amplitude recovery (see e.g. Herrmann et al., 2006, and contributions by the authors to the proceedings of this conference) and compressed wavefield extrapolation (Lin and Herrmann, 2007).

While the non-parametric curvelet-based method recovers incomplete data, the physics of wave propagation is not truely exploited. Combining the non-adaptive curvelet transform with the data-adaptive focal transform (Berkhout and Verschuur, 2006) leads to a powerful formulation where data is focused by inverting the primary operator (= a multidimensional 'convolution' with an estimate of the major primaries). During this curvelet-regularized inversion of the primary operator, $\boldsymbol{\Delta} \boldsymbol{P}$, propagation paths that include the surface are removed, yielding a more focused wavefield and hence a more compressed curvelet vector.

The focusing operator itself is derived from the data and contains an estimate for the major primaries obtained from e.g. a SRME-primary estimation procedure (Verschuur and Berkhout, 1997). In this abstract, we present a method where the focal operator is robustly inverted by curvelet regularization, i.e. by promoting sparsity in the curvelet domain. The robustness in this context refers to stability under noise and more importantly under missing traces, leading to an improved recovery for data with large percentages of traces missing. First, we briefly dis- 


\section{The focused curvelet transform}

cuss sparsity promoting inversion, followed by curvelet recovery by sparse inversion (CRSI). Next, we combine this method with the focal transform, leading to focused curvelet recovery by sparse inversion (fCRSI). The proposed algorithm is tested on a 3D seismic data volume.

\section{SPARSITY-PROMOTING INVERSION}

To exploit curvelets, incomplete and noisy measurements are related to a sparse curvelet coefficient vector, $\mathbf{x}_{0}$, according to

$$
\mathbf{y}=A \mathbf{x}_{0}+\mathbf{n}
$$

with $\mathbf{y}$ a vector with noisy and incomplete measurements; $\boldsymbol{A}$ the synthesis matrix that includes the inverse curvelet transform $\left(\boldsymbol{C}^{T}\right)$; and $\mathbf{n}$, a zero-centered white Gaussian noise. The matrix $\boldsymbol{A}$ is a wide rectangular matrix, so the vector $\mathbf{x}_{0}$ can not readily be calculated from the measurements, because there exist infinitely many vectors that match $\mathbf{y}$.

Recent work in 'compressive sampling' (Candès et al., 2006; Donoho, 2006) has shown that rectangular matrices can stably be inverted by solving a nonlinear sparsity promoting program (Elad et al., 2005). These inversions require a fast decay for the magnitude-sorted curvelet coefficients. Following these results, the vector $\mathbf{x}_{0}$ can be recovered from noise-corrupted and incomplete data. Sparsity-promoting norm-one penalty functionals are not new to the geosciences (see for instance the seminal work of Claerbout and Muir (1973), followed by many others). New are (i) the curvelet transform that obtains near optimal theoretical and empirical (Candes et al., 2006; Hennenfent and Herrmann, 2006b) compression rates on seismic data and images and (ii) the theoretical understanding of the conditions for a successful recovery.

In this work, the seismic recovery problem is solved by the norm-one nonlinear program:

$$
\mathbf{P}_{\varepsilon}: \quad\left\{\begin{array}{l}
\widetilde{\mathbf{x}}=\arg \min _{\mathbf{x}}\|\mathbf{x}\|_{1} \quad \text { s.t. } \quad\|\boldsymbol{A} \mathbf{x}-\mathbf{y}\|_{2} \leq \varepsilon \\
\widetilde{\mathbf{f}}=\boldsymbol{S}^{T} \widetilde{\mathbf{x}}
\end{array}\right.
$$

in which $\varepsilon$ is a noise-dependent tolerance level. The nonlinear program $\mathbf{P}_{\varepsilon}$ is general and the (curvelet-based) synthesis matrix, $\boldsymbol{A}$, and the inverse sparsity transform, $\boldsymbol{S}^{T}$, are defined in accordance with the application. The vector $\widetilde{\mathbf{f}}$ represents the estimated solution (denoted by the symbol ). The above nonlinear program is solved with a threshold-based cooling method following ideas from Figueiredo and Nowak (2003) and Elad et al. (2005).

\section{SEISMIC DATA RECOVERY}

\section{CRSI}

In our formulation, seismic data regularization involves the solution of $\mathbf{P}_{\varepsilon}$ with $\boldsymbol{A}:=\boldsymbol{R} \boldsymbol{C}^{T}, \boldsymbol{S}:=\boldsymbol{C}$ given incomplete data, $\mathbf{y}=\boldsymbol{R} \mathbf{f}$, with $\mathbf{f}$ the fully sampled data and $\boldsymbol{R}$ the picking matrix that selects the acquired traces from the total data volume. In recent years, the authors repeatedly reported on successful curvelet-based recovery of seismic data (see e.g. Herrmann, 2005; Hennenfent and Herrmann, 2006a, 2007). Compared to other methods, such as sparse Fourier recovery (Sacchi and Ulrych, 1996; Zwartjes and Gisolf, 2006) and plane-wave destruction (Fomel and Guitton, 2006), curvelet-based methods work for data with conflicting dips. Fig. 2 contains an example where data is recovered from $85 \%$ traces missing. This figure shows that CRSI is able to recover the complete data volume at the expense of the highest frequency band. This estimate for the interpolated data is used to calculate an improved estimate for the primaries.

\section{fCRSI}

Combining the non-adaptive curvelet transform with the data-adaptive focal transform (Berkhout and Verschuur, 2006), leads to a powerful formulation where data is focused by inverting the primary operator. During this curvelet-regularized inversion of the primary operator, $\boldsymbol{\Delta} \boldsymbol{P}$, propagation paths that include the surface are removed, yielding a more focused wavefield and hence a more compressed curvelet vector. This improved focusing is achieved by $\mathbf{P}_{\varepsilon}$ with the synthesis matrix $\boldsymbol{A}:=$ $\boldsymbol{R} \boldsymbol{\Delta} \boldsymbol{P C} C^{T}$ and inverse sparsity transform $\boldsymbol{S}^{T}:=\Delta \boldsymbol{P} \boldsymbol{C}^{T}$. The solution of $\mathbf{P}_{\varepsilon}$ now entails the inversion of $\Delta \boldsymbol{P} \boldsymbol{C}^{T}$, yielding the sparsest set of curvelet coefficients that matches the incomplete data when 'convolved' with the primaries. The symbol $\boldsymbol{\Delta} \boldsymbol{P}$ refers to applying a temporal Fourier transform, followed by a frequency-slice-by-frequencyslice matrix multiplication by $\widehat{\Delta P}(\omega)$, followed by an inverse temporal Fourier transform for each slice. This operator is compounded with the 3-D inverse curvelet transform that brings the data from the curvelet domain back to a 3D data volume. This choice for the synthesis operator corresponds to a curvelet-regularized formulation of the focal transform (Berkhout and Verschuur, 2006).

The focal transform corresponds to an imaging towards the source without applying an imaging condition. After applying the focal transform, the data is focused towards the source, a property used by Berkhout and Verschuur (2006) who "cut out" the aliased energy in the focal domain, prior to applying the inverse focal transform. In our approach, we follow a less 'ad hoc' approach by only promoting sparsity in the domain spanned by the 


\section{The focused curvelet transform}

focused curvelet transform. In this way, no assumptions except for sparsity are made. This sparsity assumption seems reasonable since curvelets are sparse on wavefields and the focused data itself is a wavefield, where the primaries are mapped to a directional source and the first-order multiples are mapped to primaries etc. etc. Because the wavefield is stripped from one interaction with the surface, the focused wavefield will be more focused and hence the sparsity-promoting norm in the curvelet domain will be more effective.

Aside from the focusing argument, the improved performance (cf. Fig 2(a) and 2(b)) can be attributed to the increase in mutual incoherence between the Dirac measurement basis and the columns of $\Delta \boldsymbol{P} \boldsymbol{C}^{T}$ (see also Herrmann and Hennenfent, 2007; Hennenfent and Herrmann, 2007). While CRSI could only recover the data volume with the finest scale removed, fCRSI is able to recover the full data leading to a sharper recovery, especially visible for the diffracted events in the time slice. The improvements for the recovery reflect in an improvement for the predicted multiples as shown in Fig. 3. In turn, the fCSRI recovered data yields an improved prediction for the multiples.

\section{DISCUSSION AND CONCLUSIONS}

The presented methodology banks on two properties of curvelets: their ability to detect wavefronts (the 'wavefront set') and their approximate invariance under wave propagation. By compounding the curvelet transform with the focal transform, we were able to improve the recovery from incomplete data by curvelet-based sparsity promotion. This improved performance is due to the additional focusing by the primaries, rendering the curvelet-sparsity promotion during the recovery more effective. As with curvelet-based recovery without focusing, the recovery is improved by random sampling. This imperative random sampling breaks the aliasing by turning the missing data into a removable noise-term. For further details on this important observation, refer to other contributions by the authors to the proceedings of this conference. Since the focal transform corresponds to an imaging of seismic data towards the source, our results suggest that migrated images can in principle be recovered from data with large percentages of random traces missing.

\section{ACKNOWLEDGMENTS}

The authors would like to thank Eric Verschuur for providing us with the dataset. We also would like to thank the authors of CurveLab for making their codes available. The examples presented were prepared with Madagascar (rsf.sourceforge. net/), supplemented by SLIMPy (s lim. eos . ubc. ca/SLIMpy) operator overloading, developed by Sean Ross Ross. This work was in part financially supported by the NSERC Discovery (22R81254) and CRD Grants DNOISE (334810-05) of F.J.H. and was carried out as part of the SINBAD project with support, secured through ITF, from BG Group, BP, Chevron, ExxonMobil and Shell.
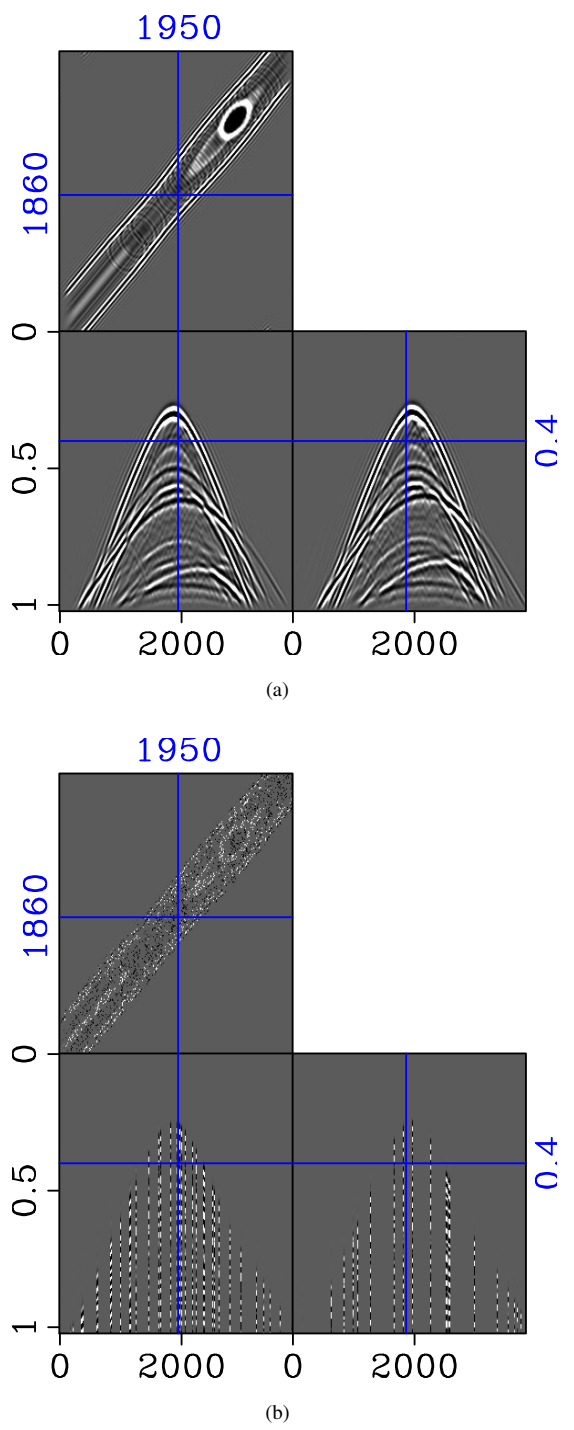

Figure 1: Synthetic dataset. (a) Original data. (b) Randomly subsampled data with $85 \%$ of the traces missing. 


\section{The focused curvelet transform}
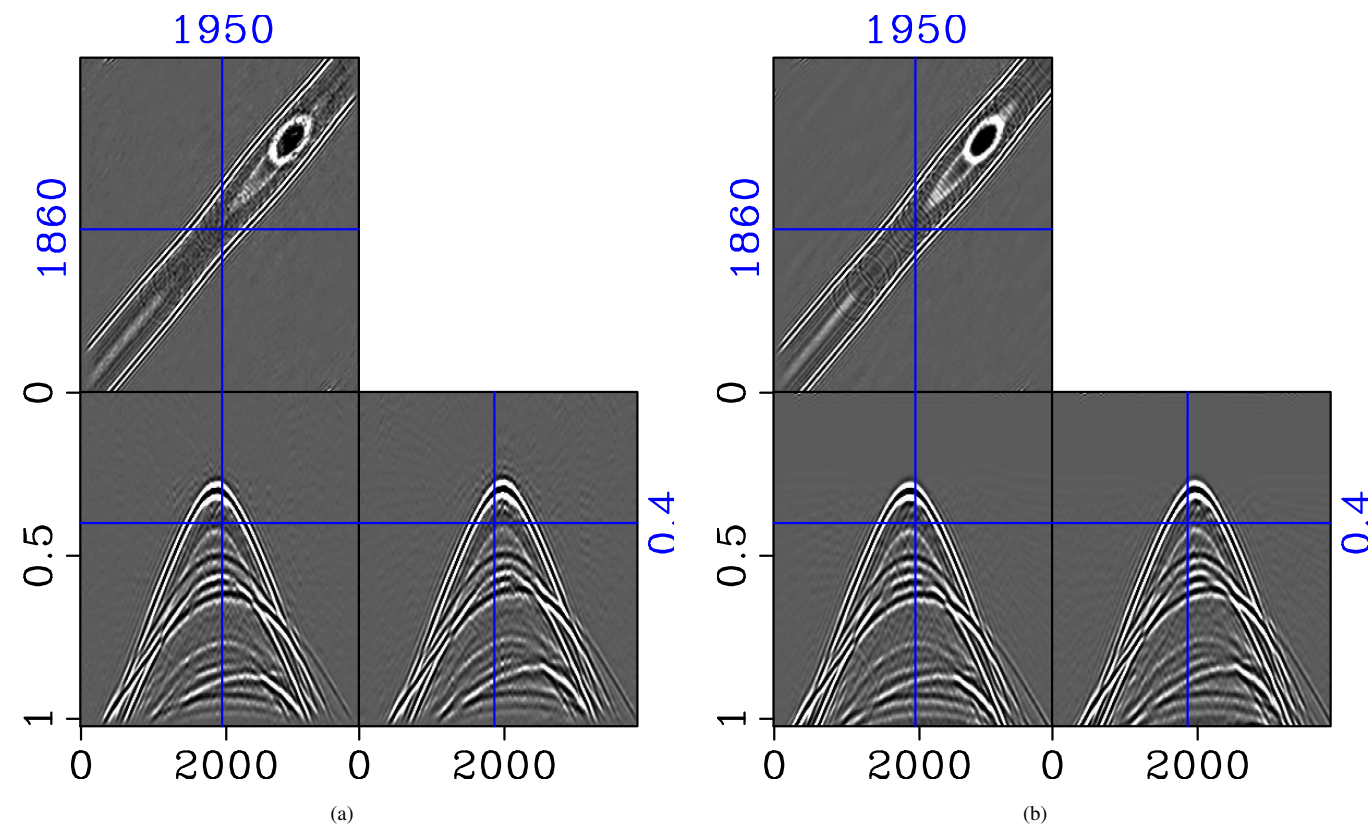

Figure 2: Curvelet-based seismic data recovery. (a) Recovery with CRSI. (b) Recovery with fCRSI. Comparison between the CRSI- and fCRSI results shows a clear improvement in the frequency content of the recovered data for fCRSI.
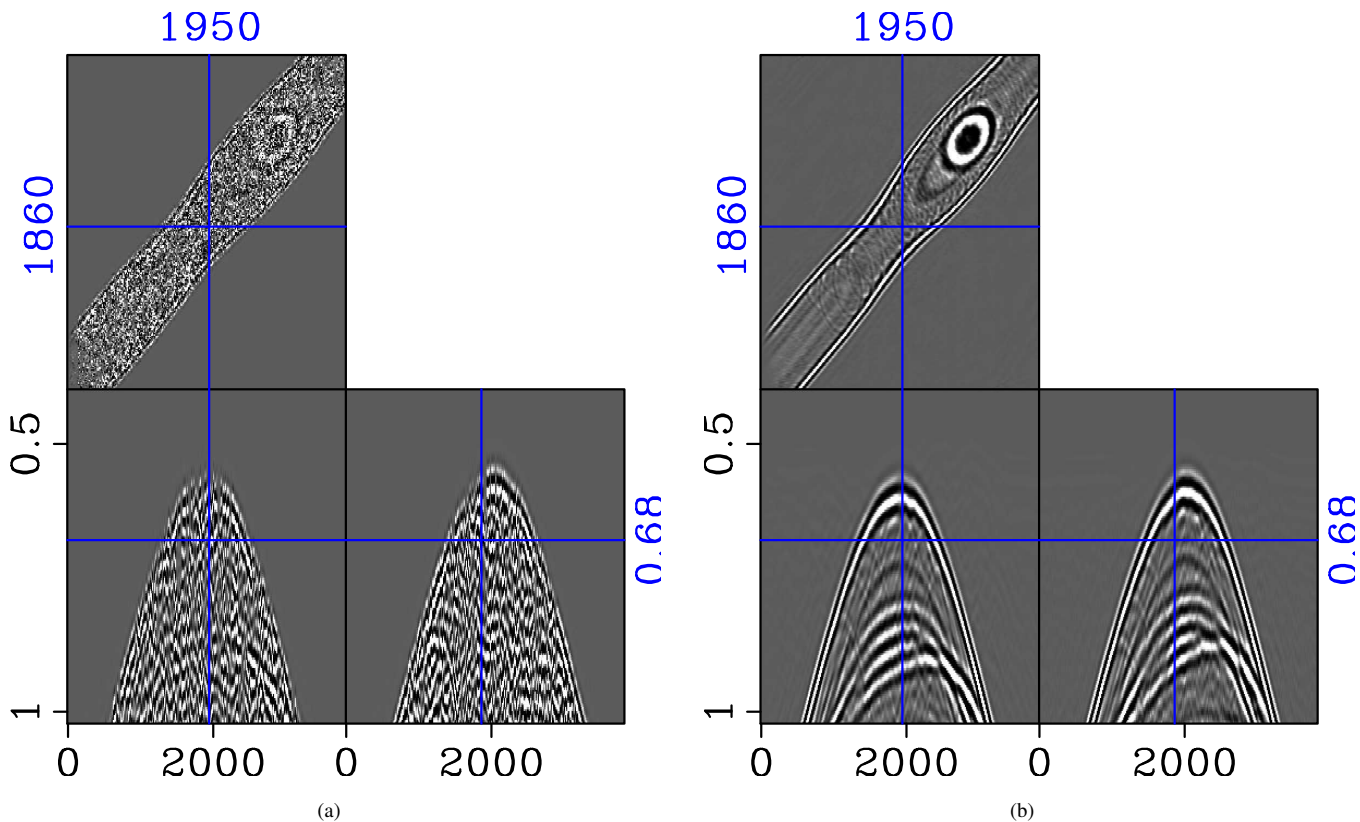

Figure 3: SRME-multiple prediction. (a) SRME-predicted multiples from randomly subsampled data with $85 \%$ of the traces missing (cf. Fig. 1(b)). (b) SRME-predicted from the fCRSI recovered data (cf. Fig. 2(b)). 


\section{The focused curvelet transform}

\section{REFERENCES}

Abma, R. and N. Kabir, 2006, 3D interpolation of irregular data with a POCS algorithm: Geophysics, 71, E91-E97.

Berkhout, A. J. and D. J. Verschuur, 2006, Focal transformation, an imaging concept for signal restoration and noise removal: Geophysics, $\mathbf{7 1 .}$

Bleistein, N., J. Cohen, and J. Stockwell, 2001, Mathematics of Multidimensional Seismic Imaging, Migration and Inversion: Springer.

Candès, E., J. Romberg, and T. Tao, 2006, Stable signal recovery from incomplete and inaccurate measurements: Comm. Pure Appl. Math., 59, 1207-1223.

Candes, E. J., L. Demanet, D. L. Donoho, and L. Ying, 2006, Fast discrete curvelet transforms: SIAM Multiscale Model. Simul., 5, 861-899.

Claerbout, J. and F. Muir, 1973, Robust modeling with erratic data: Geophysics, 38, 826-844.

Donoho, D. L., 2006, Compressed sensing: IEEE Trans. Inform. Theory, 52, 1289-1306.

Elad, M., J. L. Starck, P. Querre, and D. L. Donoho, 2005, Simulataneous Cartoon and Texture Image Inpainting using Morphological Component Analysis (MCA): Appl. Comput. Harmon. Anal., 19, 340358.

Figueiredo, M. and R. Nowak, 2003, An EM algorithm for wavelet-based image restoration: IEEE Trans. Image Processing, 12, 906-916.

Fomel, S. and A. Guitton, 2006, Regularizing seismic inverse problems by model reparameterization using plane-wave construction: Geophysics, 71, A43-A47.

Hennenfent, G. and F. Herrmann, 2006a, Application of stable signal recovery to seismic interpolation: Presented at the SEG International Exposition and 76th Annual Meeting.

$\ldots$, 2007, Irregular sampling: from aliasing to noise: Presented at the EAGE 69th Conference \& Exhibition.

Hennenfent, G. and F. J. Herrmann, 2006b, Seismic denoising with non-uniformly sampled curvelets: IEEE Comp. in Sci. and Eng., 8, 16-25.

Herrmann, F. J., 2005, Robust curvelet-domain data continuation with sparseness constraints: Presented at the EAGE 67th Conference \& Exhibition Proceedings.

Herrmann, F. J., U. Boeniger, and D.-J. E. Verschuur, 2007, Nonlinear primary-multiple separation with directional curvelet frames: Geoph. J. Int. To appear.

Herrmann, F. J. and G. Hennenfent, 2007, Non-parametric seismic data recovery with curvelet frames. Submitted for publication.

Herrmann, F. J., P. P. Moghaddam, and C. Stolk, 2006, Sparsety- and continuity-promoting seismic imaging with curvelet frames. In revision.

Lin, T. and F. J. Herrmann, 2007, Compressed wavefield extrapolation. in revision.

Sacchi, M. and T. Ulrych, 1996, Estimation of the discrete fourier transform, a linear inversion approach: Geophysics, 61, 1128-1136.

Spitz, S., 1999, Pattern recognition, spatial predictability, and subtraction of multiple events: The Leading Edge, 18, 55-58.

Verschuur, D. J. and A. J. Berkhout, 1997, Estimation of multiple scattering by iterative inversion, part II: practical aspects and examples: Geophysics, 62, 1596-1611.

Wapenaar, C., D. Draganov, and J. Robertsson, eds., 2006, Supplement Seismic Interferometry. SEG.

Ying, L., L. Demanet, and E. J. Candés, 2005, 3D discrete curvelet transform: Wavelets XI, Expanded Abstracts, 591413, SPIE.

Zwartjes, P. and A. Gisolf, 2006, Fourier reconstruction of marine-streamer data in four spatial coordinates: Geophysics, 71, V171-V186. 